Supporting Information for

\title{
Characterizing The $\mathrm{CH}_{3} \mathrm{SSCH}_{3}-\mathrm{Au}(111)$ System From Single Molecules To Full Surface Coverage - A Scanning Tunneling Microscopy Study.
}

\author{
Yi C. Zhang, David Y. Lee, K. W. Hipps* \\ Washington State University \\ Pullman, WA 99164-4630
}

Contents

Page

Figure S1. STM images of the DMDS-Au(111) system after annealing to $120 \mathrm{~K}$.

Figure S2. Color STM image of Figure $2 \mathrm{~d}$ of the manuscript.

Table S1. Number of molecules per $\AA^{2}$ for each stripe-phase regime.

Figure S3. Top view of DFT optimized structures for single-molecule DMDS (a), two possible $2 \mathrm{SCH}_{3}$ radicals $(b, c)$, and the DMDS monolayer (d).

Figure S4. Activation barrier for dissociation with structure retention.

Figure S5: Various optimized positions for two $\mathrm{SCH} 3$ radical on $\mathrm{Au}(111)$ 


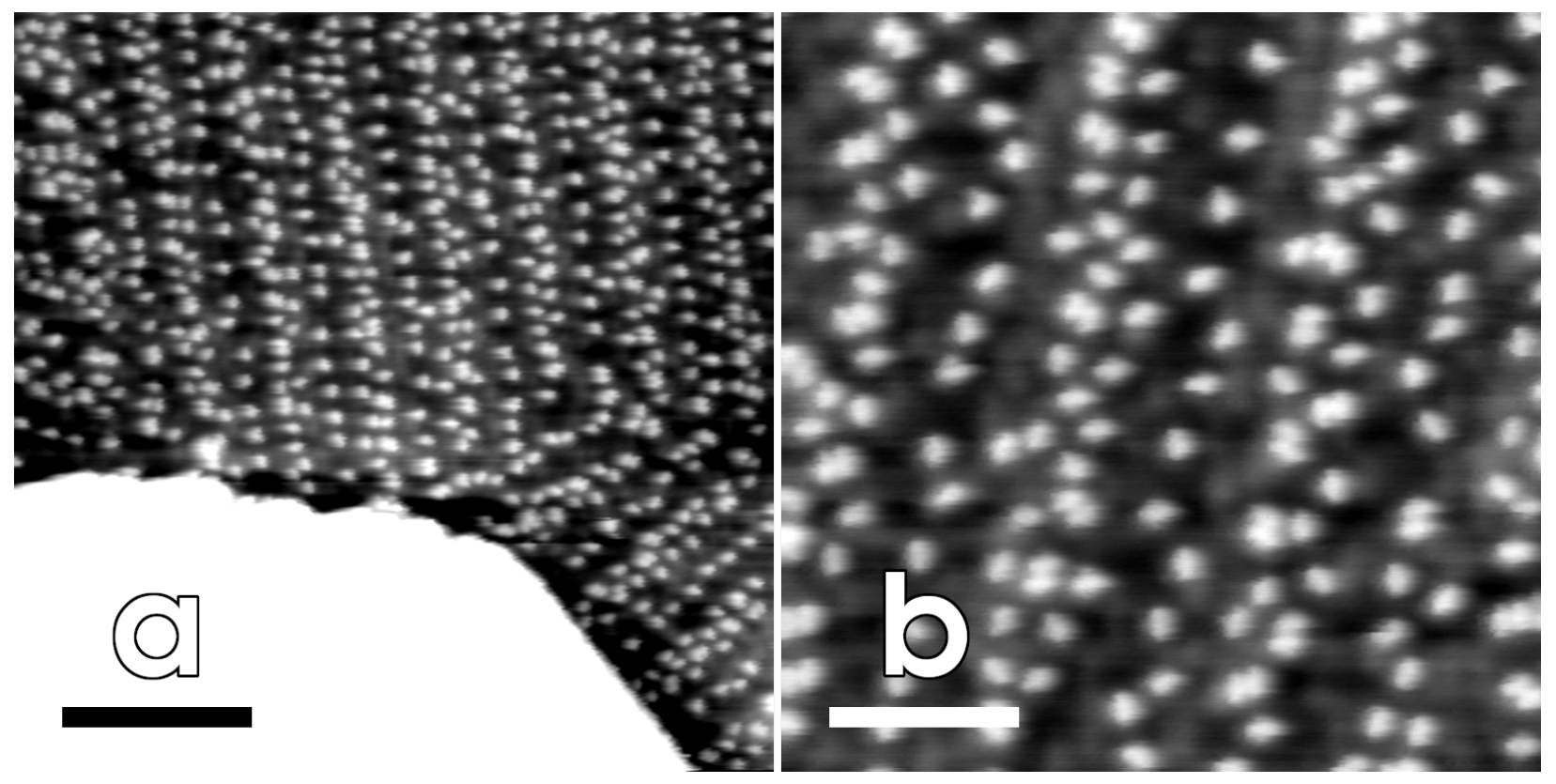

Figure S1(a), (b) STM images of the DMDS-Au(111) system after annealing to 120K. The DMDS molecules were first deposited onto a $12 \mathrm{~K}$ surface. Tunneling parameters for both images are, $500 \mathrm{mV}$ at $15 \mathrm{pA}$. The black scale bar of (a) is $125 \AA$. The white scale bar of $(b)$ is $50 \AA$.

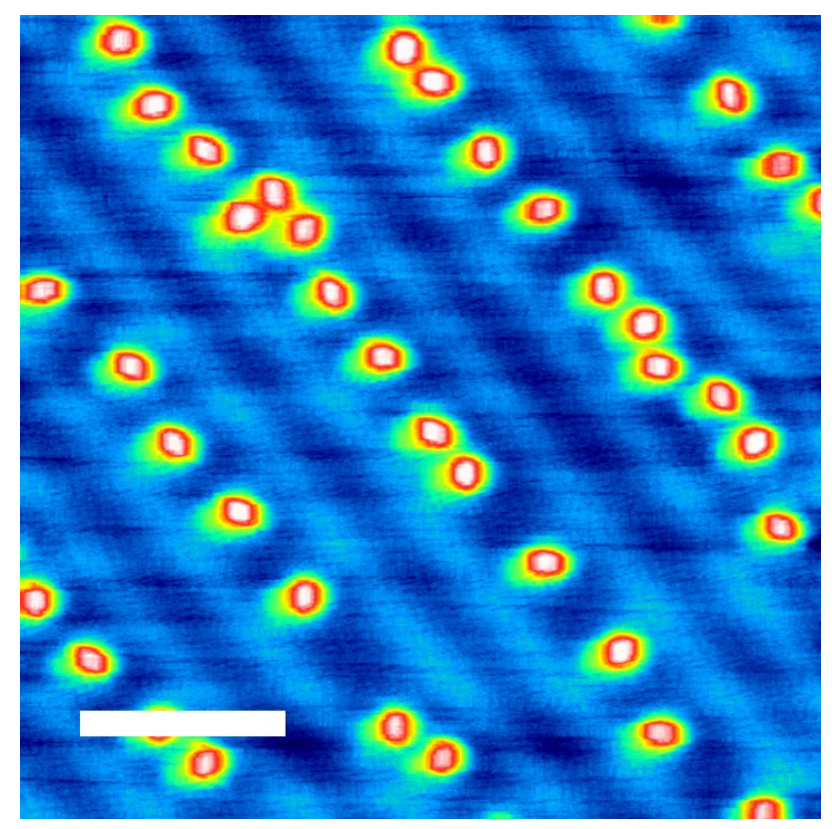

Figure S1, Colored STM image of Figure 2d of the manuscript. The white scale bar is $50 \AA$.

Table S1, Number of molecules per $\AA^{2}$ for each stripe-phase regime.

\begin{tabular}{|c|c|c|}
\hline Single-file & Double-file & Single-file and Staggered \\
\hline 0.2 molecules/ $\AA^{2}$ & 0.45 molecules $/ \AA^{2}$ & 0.65 molecules/ $\AA^{2}$ \\
\hline
\end{tabular}



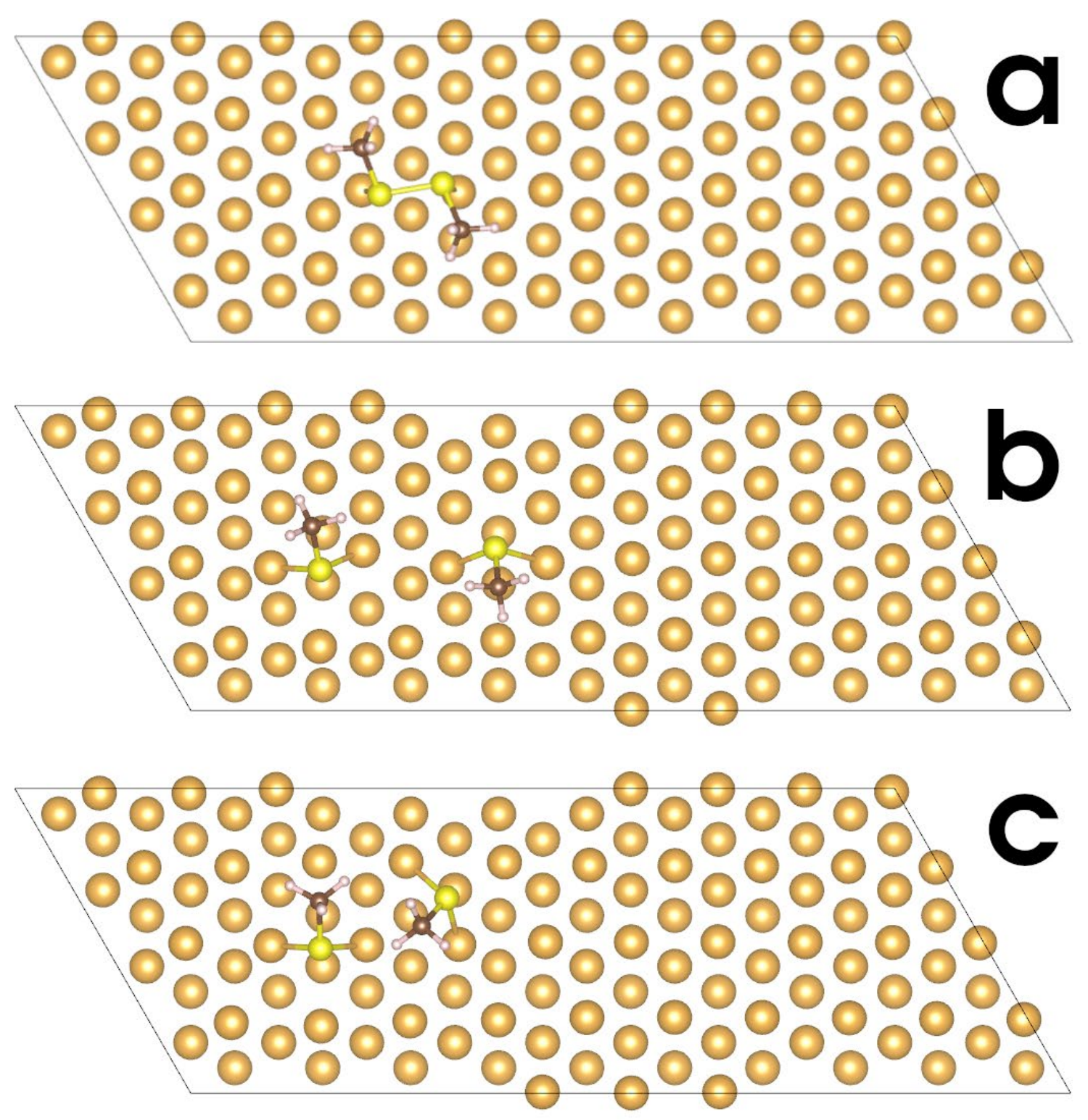

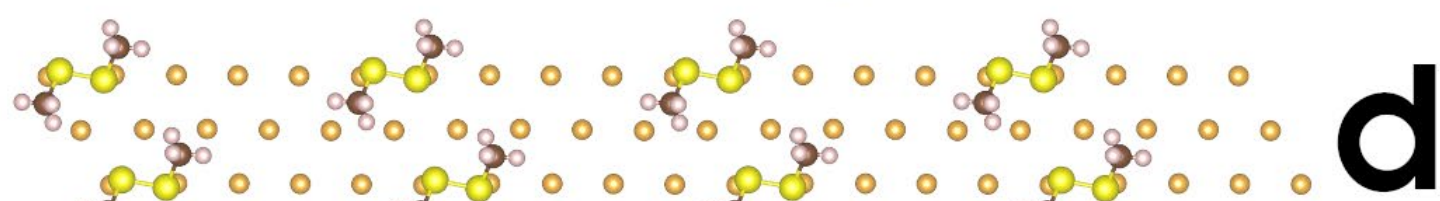

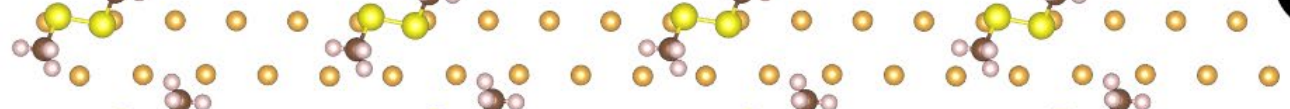

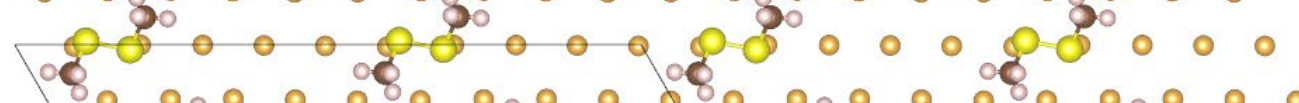

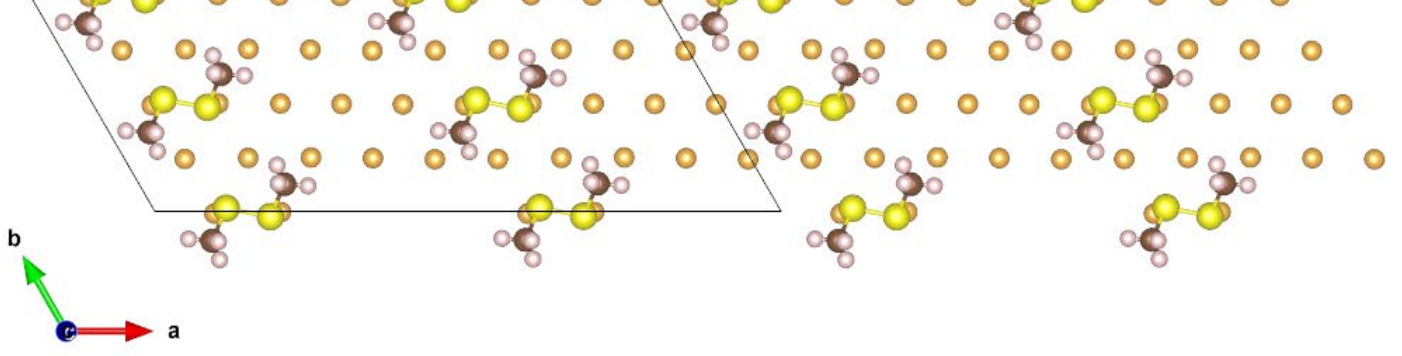

Figure S2. Top view of DFT optimized structures for single-molecule DMDS (a), two possible $2 \mathrm{SCH}_{3}$ radicals (b, $\left.c\right)$, and the DMDS monolayer (d). 

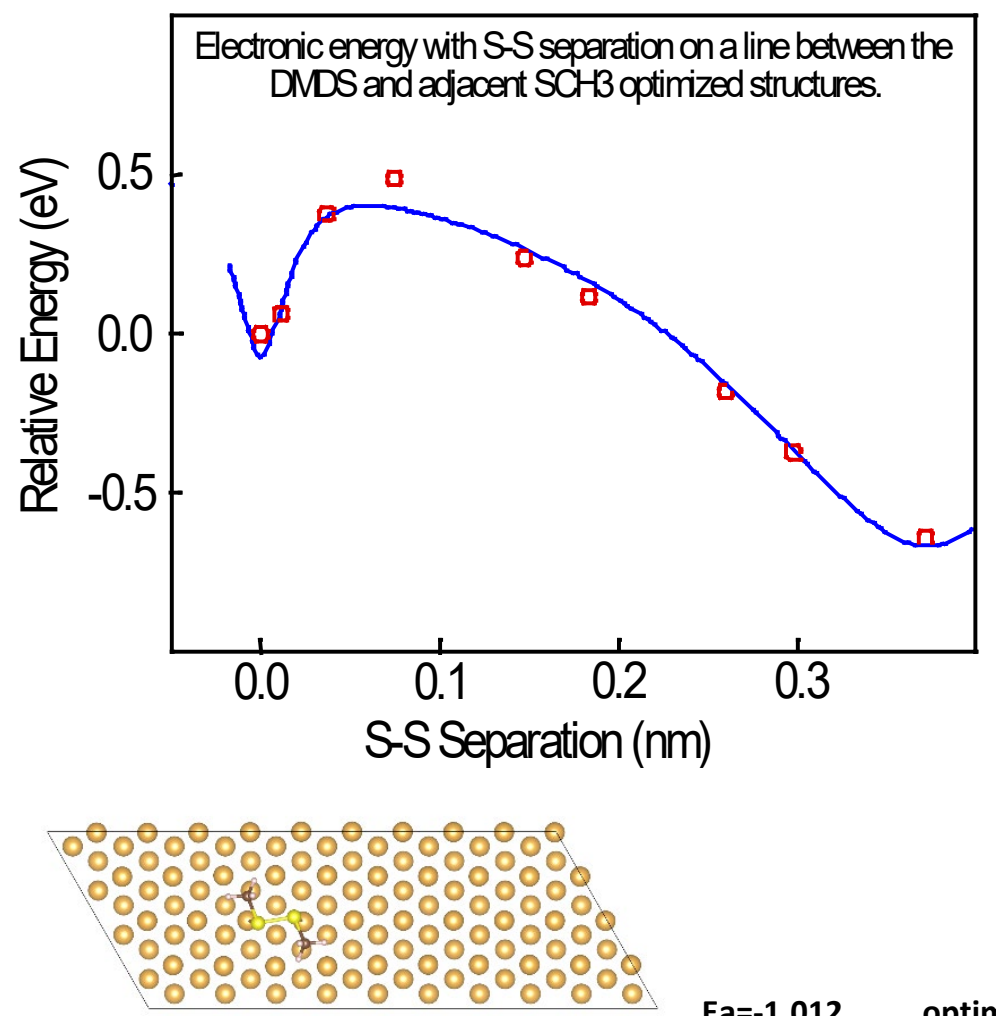

Ea=-1.012 optimized DMDS structure
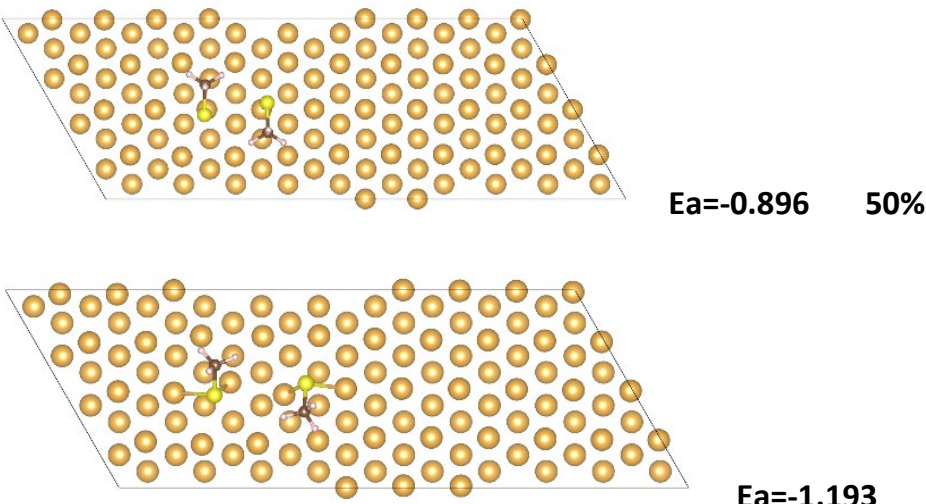

$E a=-1.193 \quad 70 \%$

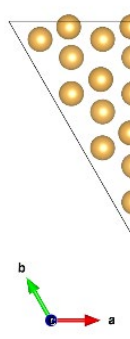

$E a=-1.565$

optimized structure of $2 \mathrm{SCH} 3$ radicals

Figure S3. Activation barrier for dissociation with structure retention. Electronic adsorption energies and optimized structures computed for several different configurations and separations of two methanesulfide radicals on a 3 layer $4 \times 10$ Au(111) lattice (only the top layer of Au is shown). The intermediate positions were chosen along a line connecting $S$ atom positions between the minimum energy DMDS and two radical positions. In each intermediate structure $C, H$, and Au top layer positions were optimized. The smooth curve is a best fit of a superposition of Morse potential functions. 


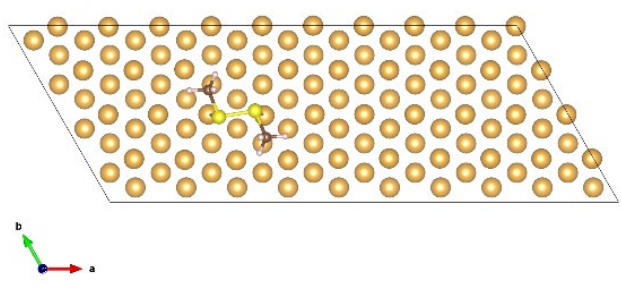

\section{Ea=-1.012 optimized DMDS minimum energy structure}

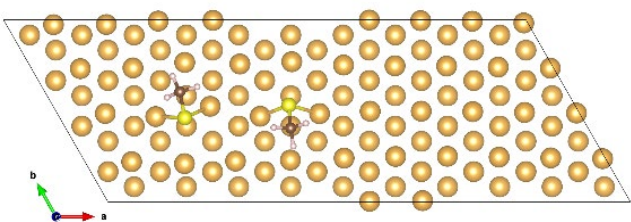

$\mathrm{Ea}=-\mathbf{1 . 5 6 5}$ Au_SC_4x10_2K1

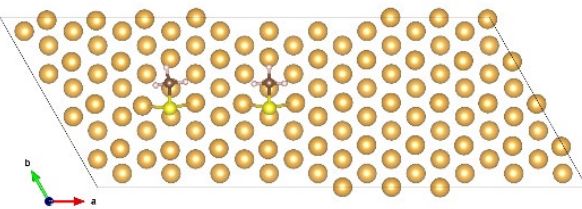

$E a=-1.438$ Au_SC_4x10_2K1B

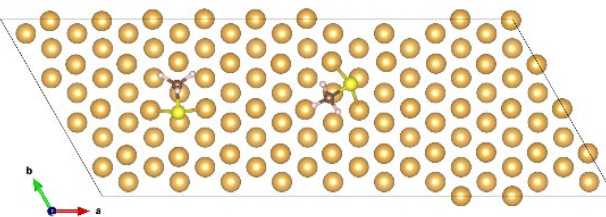

$E a=-1.368$ Au_SC_4x10_2K1E

$1000.000 .0 \% 0.000$

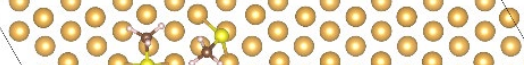

\%० -0.\%:\%:\%:\%:\%

$\longrightarrow . .0 \% 0 \% 0 \%$

$E a=-1.359 \quad A u \_S C \_4 \times 10 \_2 K 1 C$

Figure S4. Various optimized positions for two SCH3 radical on Au(111). For all these optimizations only the lower two layers of Au(111) were fixed in position. Note that the radicals have cooperative interactions - they have lower energy close to each other than far away. 
CONTCAR file for the Optimized SAM

$\mathrm{Au} \mathrm{C} \mathrm{H} \mathrm{S}$

1.00000000000000

28.8375820000000012

$-5.7675165000000002$

0.0000000000000000

9.9896316100000000

0.0000000000000000

0.0000000000000000

0.0000000000000000

23.0637359999999987

$\begin{array}{llllll}\mathrm{Au} & \mathrm{C} & \mathrm{H} & \mathrm{S} & \\ 120 & & 8 & & 24 & 8\end{array}$

Selective dynamics

Direct

$0.0666670000950020 \quad 0.0833329999040018$

$0.7008446142464495-0.0021906336541027$

$0.5932223944021190 \quad 0.9905973472386345$

$0.4946804326305304 \quad 0.9935457848307914$

$0.3972448178267218 \quad 0.9958414116075972$

$0.2999924078500026 \quad 0.9977572858804216$

$0.2007690273071409-0.0019175303144842$

$0.0931425804340537 \quad 0.9906676287461030$

$0.9946554202513049 \quad 0.9936127154508105$

$\begin{array}{lll}0.9333329999049980 & 0.9166670000959982\end{array}$

$0.7333329999050022 \quad 0.9166670000959982$

$0.6333329999050008 \quad 0.9166670000959982$

$0.8000015297807461 \quad 0.9975764158955609$

$\begin{array}{lll}0.1333330000390021 & 0.6666666670000012\end{array}$

$0.5333329999049994 \quad 0.9166670000959982$

$0.2333329999050022 \quad 0.9166670000959982$

$0.1333329999050008 \quad 0.9166670000959982$

$\begin{array}{lll}0.0333329999049994 & 0.9166670000959982\end{array}$

$0.9333330000389992 \quad 0.6666666670000012$

$0.8333333332840027 \quad 0.6666666670000012$

$0.7333330000390035 \quad 0.6666666670000012$

$0.6333330000390021 \quad 0.6666666670000012$

$0.5333330000390006 \quad 0.6666666670000012$

$0.4333330000389992 \quad 0.6666666670000012$

$0.3333333332840027 \quad 0.6666666670000012$

$0.2333330000390035 \quad 0.6666666670000012$

$0.4333329999049980 \quad 0.9166670000959982$

$0.3333333334970021 \quad 0.9166670000959982$

$0.8971947948106033 \quad 0.9956804056233032$

$\begin{array}{lll}0.8333333334970021 & 0.9166670000959982\end{array}$

$0.6983181440533619 \quad 0.7468926137114258$

$0.4946021208575832 \quad 0.4938608823377972$

$0.5960975885124695 \quad 0.7425332536681092$

$\begin{array}{lll}0.4953261858215327 & 0.7412681791747209\end{array}$

$0.3973290955249342 \quad 0.7461260986374977$

0.2991354186107718

0.1983517361584782

0.0960514716480632

0.9952388429599441

0.8971989907150375

0.7998921319305182

0.7005883975498925

0.5929700215223377

0.9952052947520897

0.2999707651597398

0.0961409297962631

0.2007495105120507

0.0930220853797671

0.9945949802391211

0.8973058033906494

0.7991710363149667

0.6984503977108594

0.5959468175911697

0.7494075947668182

0.7473334906757356

0.7423403001963262

0.7410682291040749

0.4959046573912008

0.4977048549853840

0.4978038441213528

0.4905705803901994

0.2408071514701522

0.4977485775860918

0.2424124386414774

0.4979611029303449

0.4903780116166126

0.4937223169733523

0.2460875895510869

0.2494418272222794

0.2474112043847546

0.2420806615607624

0.4951734472582728

0.2408450821338522

0.3972970501978723

0.2991292340806759

0.2461604425032300

0.2494700101686365

0.1020900000759966

0.3080264984482526

0.3088463045925272

0.3111807709252424

0.3118345274238876

0.3108886474211158

0.3080969713361900

0.3088250169730619

0.3111205467919196

0.2041800001530021

0.2041800001530021

0.2041800001530021

0.3108364450099891

0.2041800001530021

0.2041800001530021

0.2041800001530021

0.2041800001530021

0.2041800001530021

0.2041800001530021

0.2041800001530021

0.2041800001530021

0.2041800001530021

0.2041800001530021

0.2041800001530021

0.2041800001530021

0.2041800001530021

0.2041800001530021

0.2041800001530021

0.3117845945809565

0.2041800001530021

0.3053434037585994

0.3110834985316366

0.3055470772676949

0.3098824758794593

0.3112827998175368

0.3093538934118165

0.3051827852892604

0.3054620429836364

0.3097775740896146

0.3118049440613989

0.3108935694854325

0.3081379714611441

0.3087326101145613

0.3097474701299752

0.3108867200168599

0.3055266342088609

0.3081836366451361

0.3087708445965680

0.3111071767100845

0.3112829250379364

0.3093325648907399

0.3051695890390184

0.3054316321745706

0.3096914335755246

0.3112942238165524

0.3094158916587439

S6 
0.1983802975435810

0.3972548703700301

0.0333330000390006

0.8666670000949992

0.9333329998050033

0.6666666666029997

0.4666669999609994

0.5666670001949967

0.4666670001950024

0.3666670001950010

0.2666670001949996

0.1666666666029997

0.0666670001949967

0.9666669999609994

0.8666669999609979

0.7666669999609965

0.6666666667159973

0.5666669999610008

0.7666670001949996

0.2666669999609965

0.9666670001950024

0.1666666667159973

0.0666669999610008

0.8333333333970003

0.7666670000949978

0.6666666665029979

0.5666670000950020

0.4666670000950006

0.3666670000949992

0.2666670000949978

0.1666666665029979

0.3666669999609979

0.8666670001950010

0.0666670000610026

0.7333329998050004

0.9666670000950006

0.8972410005979302

0.5333329998049976

0.4333329998050033

0.3333333333970003

0.2333329998050004

0.1333329998049990

0.0333329998049976

0.9333329999389974

0.8333333331840009

0.7333329999390017

0.6333329999390003

0.5333329999389989

0.4333329999389974

0.6333329998049990

0.3333333331840009

0.1333329999390003

0.0333329999389989

0.9666670000610011

0.8666670000609997

0.7666670000609983

0.6666666668159991

0.5666670000610026

0.4666670000610011

0.3666670000609997

0.2666670000609983

0.1666666668159991

0.7989954655571422

0.2333329999390017

0.0645352285015895

0.2319529178885351
0.2473908857544026

0.4959985803796342

0.6666666670000012

0.0833329999040018

0.4166669995950016

0.5833330004049984

0.3333333329999988

0.5833330004049984

0.5833330004049984

0.5833330004049984

0.5833330004049984

0.5833330004049984

0.5833330004049984

0.3333333329999988

0.3333333329999988

0.3333333329999988

0.3333333329999988

0.3333333329999988

0.5833330004049984

0.3333333329999988

0.5833330004049984

0.3333333329999988

0.3333333329999988

0.4166669995950016

0.0833329999040018

0.0833329999040018

0.0833329999040018

0.0833329999040018

0.0833329999040018

0.0833329999040018

0.0833329999040018

0.3333333329999988

0.5833330004049984

0.8333333335000006

0.4166669995950016

0.0833329999040018

0.7460552851482225

0.4166669995950016

0.4166669995950016

0.4166669995950016

0.4166669995950016

0.4166669995950016

0.4166669995950016

0.1666666664999994

0.1666666664999994

0.1666666664999994

0.1666666664999994

0.1666666664999994

0.1666666664999994

0.4166669995950016

0.1666666664999994

0.1666666664999994

0.1666666664999994

0.8333333335000006

0.8333333335000006

0.8333333335000006

0.8333333335000006

0.8333333335000006

0.8333333335000006

0.8333333335000006

0.8333333335000006

0.8333333335000006

0.7490849562170100

0.1666666664999994

0.3655473977346794

0.1260550813008968
0.3053139128977935

0.3117943496586492

0.2041800001530021

0.1020900000759966

0.2041800001530021

0.1020900000759966

0.1020900000759966

0.1020900000759966

0.1020900000759966

0.1020900000759966

0.1020900000759966

0.1020900000759966

0.1020900000759966

0.1020900000759966

0.1020900000759966

0.1020900000759966

0.1020900000759966

0.1020900000759966

0.1020900000759966

0.1020900000759966

0.1020900000759966

0.1020900000759966

0.1020900000759966

0.2041800001530021

0.1020900000759966

0.1020900000759966

0.1020900000759966

0.1020900000759966

0.1020900000759966

0.1020900000759966

0.1020900000759966

0.1020900000759966

0.1020900000759966

0.1020900000759966

0.2041800001530021

0.1020900000759966

0.3112696637173191

0.2041800001530021

0.2041800001530021

0.2041800001530021

0.2041800001530021

0.2041800001530021

0.2041800001530021

0.2041800001530021

0.2041800001530021

0.2041800001530021

0.2041800001530021

0.2041800001530021

0.2041800001530021

0.2041800001530021

0.2041800001530021

0.2041800001530021

0.2041800001530021

0.1020900000759966

0.1020900000759966

0.1020900000759966

0.1020900000759966

0.1020900000759966

0.1020900000759966

0.1020900000759966

0.1020900000759966

0.1020900000759966

0.3095203593038169

0.2041800001530021

0.4532448398777182

0.4531509241791047

\begin{tabular}{|c|c|}
\hline $\mathrm{T}$ & $\mathrm{T}$ \\
\hline $\mathrm{T}$ & $\mathrm{T}$ \\
\hline $\mathrm{F}$ & $\mathrm{F}$ \\
\hline $\mathrm{F}$ & $\mathrm{F}$ \\
\hline $\mathrm{F}$ & $\mathrm{F}$ \\
\hline$F$ & $\mathrm{~F}$ \\
\hline $\mathrm{F}$ & $\mathrm{F}$ \\
\hline $\mathrm{F}$ & $\mathrm{F}$ \\
\hline $\mathrm{F}$ & $\mathrm{F}$ \\
\hline $\mathrm{F}$ & $\mathrm{F}$ \\
\hline $\mathrm{F}$ & $\mathrm{F}$ \\
\hline $\mathrm{F}$ & $\mathrm{F}$ \\
\hline$F$ & $\mathrm{~F}$ \\
\hline$F$ & $\mathrm{~F}$ \\
\hline$F$ & $\mathrm{~F}$ \\
\hline $\mathrm{F}$ & $\mathrm{F}$ \\
\hline $\mathrm{F}$ & $\mathrm{F}$ \\
\hline $\mathrm{F}$ & $\mathrm{F}$ \\
\hline $\mathrm{F}$ & $\mathrm{F}$ \\
\hline $\mathrm{F}$ & $\mathrm{F}$ \\
\hline$F$ & $\mathrm{~F}$ \\
\hline$F$ & $\mathrm{~F}$ \\
\hline $\mathrm{F}$ & $\mathrm{F}$ \\
\hline $\mathrm{F}$ & $\mathrm{F}$ \\
\hline$F$ & $\mathrm{~F}$ \\
\hline $\mathrm{F}$ & $\mathrm{F}$ \\
\hline$F$ & $\mathrm{~F}$ \\
\hline $\mathrm{F}$ & $\mathrm{F}$ \\
\hline $\mathrm{F}$ & $\mathrm{F}$ \\
\hline $\mathrm{F}$ & $\mathrm{F}$ \\
\hline$F$ & $\mathrm{~F}$ \\
\hline$F$ & $\mathrm{~F}$ \\
\hline $\mathrm{F}$ & $\mathrm{F}$ \\
\hline $\mathrm{F}$ & $\mathrm{F}$ \\
\hline$F$ & $\mathrm{~F}$ \\
\hline $\mathrm{F}$ & $\mathrm{F}$ \\
\hline $\mathrm{T}$ & $\mathrm{T}$ \\
\hline$F$ & $\mathrm{~F}$ \\
\hline$F$ & $\mathrm{~F}$ \\
\hline$F$ & $\mathrm{~F}$ \\
\hline $\mathrm{F}$ & $\mathrm{F}$ \\
\hline $\mathrm{F}$ & $\mathrm{F}$ \\
\hline $\mathrm{F}$ & $\mathrm{F}$ \\
\hline $\mathrm{F}$ & $\mathrm{F}$ \\
\hline $\mathrm{F}$ & $\mathrm{F}$ \\
\hline$F$ & $\mathrm{~F}$ \\
\hline$F$ & $\mathrm{~F}$ \\
\hline$F$ & $\mathrm{~F}$ \\
\hline $\mathrm{F}$ & $\mathrm{F}$ \\
\hline $\mathrm{F}$ & $\mathrm{F}$ \\
\hline$F$ & $\mathrm{~F}$ \\
\hline$F$ & $\mathrm{~F}$ \\
\hline $\mathrm{F}$ & $\mathrm{F}$ \\
\hline$F$ & $\mathrm{~F}$ \\
\hline $\mathrm{F}$ & $\mathrm{F}$ \\
\hline $\mathrm{F}$ & $\mathrm{F}$ \\
\hline$F$ & $\mathrm{~F}$ \\
\hline$F$ & $\mathrm{~F}$ \\
\hline $\mathrm{F}$ & $\mathrm{F}$ \\
\hline $\mathrm{F}$ & $\mathrm{F}$ \\
\hline$F$ & $\mathrm{~F}$ \\
\hline $\mathrm{F}$ & $\mathrm{F}$ \\
\hline $\mathrm{T}$ & $\mathrm{T}$ \\
\hline$F$ & $\mathrm{~F}$ \\
\hline $\mathrm{T}$ & $\mathrm{T}$ \\
\hline $\mathrm{T}$ & $\mathrm{T}$ \\
\hline
\end{tabular}

\section{S7}


0.2315397640551087

0.0645234037861650

0.7304861618489674

0.7327917943994720

0.5655401946311965

0.5632388287100977

0.0736935179617880

0.0280440000919986

0.2680824711949673

0.2223479089251308

0.2365658308503763

0.2229566718315300

0.0587876108617116

0.0283968036117980

0.2680277706465538

0.0738412543233463

0.0593755691775011

0.2376838804060170

0.7363468904105628

0.5747929525608714

0.7213617678282749

0.5293856973513076

0.7688455824440457

0.7238057565667547

0.5597997791233351

0.5724561254283831

0.5572790418449440

0.5272023211880688

0.7665569867498904

0.7385841832202269

0.6156395351270690

0.6802910262715592

0.1795172932462134

0.1168046328816946

0.1798677295716625

0.1162248587732380

0.6781649560766502

0.6177054353159772
0.6322517691328926

0.8656250782977879

0.6228634267626793

0.1291636866651904

0.8694723058069699

0.3639323194377814

0.8616578889589142

0.3712758456226152

0.6266006372016626

0.6372979946064180

0.7186574486195838

0.1302334159095504

0.7777718226814427

0.8730654649722875

0.1184341021170367

0.3627269502403133

0.2778818371284885

0.2139202179038505

0.7109656527066021

0.8665620709245685

0.6266740121837017

0.8767371433405077

0.1213628725654639

0.1342364541451766

0.7811274077258719

0.3599234605122906

0.2758536085018948

0.3718412928200023

0.6152036353245218

0.2166376674787877

0.5132210401007371

0.9790155716827850

0.9764069895751584

0.0152792267472111

0.4803173204826722

0.5157040689261162

0.4736963801480500

0.0182389152445256
0.4528718966237856

0.4533367864947518

0.4534917666256317

0.4528957931467277

0.4537068075544886

0.4533913990308465

0.4988388306099319

0.4485053524460120

0.4486138790112328

0.4982706510621743

0.4283441468844249

0.4986948748738831

0.4294698005219729

0.4487798726192622

0.4484350326935332

0.4987967804148989

0.4296772880722581

0.4292982677093926

0.4297541562103513

0.4992610030787865

0.4990143996576242

0.4489713000666828

0.4484353643641729

0.4983658543953242

0.4302820181288115

0.4988746611153180

0.4295488700104355

0.4489403830910882

0.4488702456581313

0.4286626886000889

0.4197957618469643

0.4199644018016768

0.4198316470503884

0.4198293660326411

0.4202051630838924

0.4194532784793122

0.4198285493704074

0.4196070736041602

$\begin{array}{lll}\mathrm{T} & \mathrm{T} & \mathrm{T} \\ \mathrm{T} & \mathrm{T} & \mathrm{T} \\ \mathrm{T} & \mathrm{T} & \mathrm{T} \\ \mathrm{T} & \mathrm{T} & \mathrm{T} \\ \mathrm{T} & \mathrm{T} & \mathrm{T} \\ \mathrm{T} & \mathrm{T} & \mathrm{T} \\ \mathrm{T} & \mathrm{T} & \mathrm{T} \\ \mathrm{T} & \mathrm{T} & \mathrm{T} \\ \mathrm{T} & \mathrm{T} & \mathrm{T} \\ \mathrm{T} & \mathrm{T} & \mathrm{T} \\ \mathrm{T} & \mathrm{T} & \mathrm{T} \\ \mathrm{T} & \mathrm{T} & \mathrm{T} \\ \mathrm{T} & \mathrm{T} & \mathrm{T} \\ \mathrm{T} & \mathrm{T} & \mathrm{T} \\ \mathrm{T} & \mathrm{T} & \mathrm{T} \\ \mathrm{T} & \mathrm{T} & \mathrm{T} \\ \mathrm{T} & \mathrm{T} & \mathrm{T} \\ \mathrm{T} & \mathrm{T} & \mathrm{T} \\ \mathrm{T} & \mathrm{T} & \mathrm{T} \\ \mathrm{T} & \mathrm{T} & \mathrm{T} \\ \mathrm{T} & \mathrm{T} & \mathrm{T} \\ \mathrm{T} & \mathrm{T} & \mathrm{T} \\ \mathrm{T} & \mathrm{T} & \mathrm{T} \\ \mathrm{T} & \mathrm{T} & \mathrm{T} \\ \mathrm{T} & \mathrm{T} & \mathrm{T} \\ \mathrm{T} & \mathrm{T} & \mathrm{T} \\ \mathrm{T} & \mathrm{T} & \mathrm{T} \\ \mathrm{T} & \mathrm{T} & \mathrm{T} \\ \mathrm{T} & \mathrm{T} & \mathrm{T} \\ \mathrm{T} & \mathrm{T} & \mathrm{T} \\ \mathrm{T} & \mathrm{T} & \mathrm{T} \\ \mathrm{T} & \mathrm{T} & \mathrm{T} \\ \mathrm{T} & \mathrm{T} & \mathrm{T} \\ \mathrm{T} & \mathrm{T} & \mathrm{T} \\ \mathrm{T} & \mathrm{T} & \mathrm{T} \\ \mathrm{T} & \mathrm{T} & \mathrm{T} \\ \mathrm{T} & \mathrm{T} & \mathrm{T} \\ \mathrm{T} & \mathrm{T} & \mathrm{T} \\ & & \end{array}$

